\title{
Persiguiendo con imparcialidad "el total desprecio a la Constitución": el léxico valorativo en la Querella del Fiscal de Cataluña contra Carme Forcadell i Lluís
}

\author{
Impartially prosecuting "the total contempt for the Constitution": \\ Evaluative lexis in the criminal complaint filed by the Public Prosecutor \\ of Catalonia against Carme Forcadell i Lluís
}

\section{Giovanni Garofalo}

Università degli Studi di Bergamo. giovanni.garofalo@unibg.it Recibido: 29/04/2017. Aceptado: 16/10/2017

Resumen: Se propone un estudio semántico-discursivo de las dos querellas presentadas por la Fiscalía Superior de Cataluña contra D. ${ }^{a}$ Carme Forcadell i Lluís, presidenta del Parlamento de Cataluña, y contra los miembros de la Mesa del Parlamento catalán por los delitos de desobediencia y prevaricación. Compaginando las metodologías del análisis de sentimiento, de la lingüística del corpus y de la teoría de la valoración, este estudio desmiente la idea de que la querella solicita de forma imparcial la aplicación de normas generales a casos concretos. Lejos de ser fácticos o ideacionales, los enunciados del fiscal están cargados de significados interpersonales y manifiestan implicación subjetiva con una vehemencia aledaña de la invectiva política.

Palabras clave: querella; análisis de sentimiento; polaridad textual; subjetividad; teoría de la valoración.

\begin{abstract}
This paper proposes a semantic-discursive study of the two criminal complaints filed by the Public Prosecutor of Catalonia against Mrs. Carme Forcadell i Lluís, President of the Catalan Parliament, and against key members of the Catalan Parliament's Bureau for the crimes of disobedience and misconduct. Combining sentiment analysis, corpus linguistics and appraisal theory, this study denies the idea according to which a criminal complaint seeks the application of general norms to concrete cases in an objective fashion. Far from being factual or ideational, the Prosecutor's utterances are laden with interpersonal meanings and reveal subjective implication with a vehemence reminiscent of political invective.
\end{abstract}

Keywords: criminal complaint; sentiment analysis; text polarity; subjectivity; appraisal theory.

》 Garofalo, Giovanni. 2017. "Persiguiendo con imparcialidad 'el total desprecio a la Constitución’: el léxico valorativo en la Querella del Fiscal de Cataluña contra Carme Forcadell i Lluís". Quaderns de Filologia: Estudis Lingüístics 22: 79103. doi: $10.7203 / \mathrm{qf} .22 .11302$ 



\section{El escenario discursivo de las querellas}

El 19 de octubre de 2016 el Fiscal Superior de Cataluña presentó, ante el Tribunal Superior de Justicia de Cataluña (TSJC), una querella contra D. ${ }^{a}$ Carme Forcadell i Lluís, presidenta del Parlament, acusándola de los delitos de prevaricación y de desobediencia al Tribunal Constitucional (TC), ya que había permitido que la Cámara catalana votara la hoja de ruta independentista.

El texto del fiscal precisaba que, en fecha 20 de julio de 2016, la Mesa del Parlamento autonómico, tras escuchar a la Junta de Portavoces, había tomado nota de las conclusiones de la Comisión de Estudio del Proceso Constituyente (CEPC), en las que se indicaba el recorrido hacia la desconexión. Dichas conclusiones ya habían sido declaradas inconstitucionales por un auto del TC, debidamente notificado a los querellados. Aunque los servicios jurídicos de la cámara se pronunciaran en contra de las indicaciones de la CEPC por no ser constitucionalmente admisibles, en palabras del Fiscal, Forcadell

pese a ser consciente de que tal decisión contravenía frontalmente [...] el Auto de 19 de julio de 2016, acordó someter a votación la alteración del orden del día para incluir la votación sobre las conclusiones de la Comisión de Estudio del Proceso Constituyente, resultando aprobada la alteración del orden del día y la inclusión del nuevo punto.

El 23 de febrero de 2017, la Fiscalía volvió a querellarse contra la presidenta del Parlament y los miembros soberanistas de la Mesa Lluís Corominas, Anna Simó y Ramona Barrufet por desobediencia y prevaricación, por permitir la aprobación de dos resoluciones presentadas por Junts pel Sí y la CUP que instaban a convocar un referéndum unilateral de secesión.

El sentido común prescribiría que ambos documentos judiciales se ciñeran a los hechos documentales, acudiendo a enunciados neutrales desde el punto de vista interpersonal y, por ello, "fácticos" u "objetivos". Nos esperaríamos, por ende, que el Ministerio Fiscal actuara

\footnotetext{
* Este trabajo se enmarca en el proyecto Discurso jurídico y claridad comunicativa. Análisis contrastivo de sentencias españolas y de sentencias en español del Tribunal de Justicia de la Unión Europea (Referencia FFI2015-70332-P), financiado por el Ministerio de Economía y Competitividad de España así como por los Fondos FEDER..
} 
como boca inanimada de la ley (bouche de loi) y que sus enunciados vehicularan significados que, en la terminología de la gramática sistémico-funcional, se definen como ideacionales (Halliday \& Hasan 1985: 20 ), es decir, relacionados con la mera representación de los hechos del mundo, tal como los entendemos a través de la experiencia. Este comportamiento verbal sería el más congruente con los principios de imparcialidad y objetividad que deberían guiar la actuación del Ministerio Público, según el art. 124.1 de la Constitución Española, desarrollado en el art. 7 del Estatuto Orgánico del Ministerio Fiscal (EOMF) en los términos siguientes:

Por el principio de imparcialidad el Ministerio Fiscal actuará con plena objetividad e independencia en defensa de los intereses que le estén encomendados.

Así pues, el fiscal debería ser neutral en la evaluación de los hechos y pruebas que dan lugar a la causa, sin perjudicar a ninguno de los que intervienen en el proceso, dado que su actuación ha de ser desinteresada y desapasionada, debiendo atenerse únicamente a la realidad objetiva.

Analizadas más de cerca, sin embargo, las elecciones léxicas y gramaticales del emisor resultan cargadas de significados interpersonales, que no se limitan a representar la realidad sino que interactúan con la parte contraria, a través de un amplio abanico de valoraciones subjetivas de polaridad negativa. De esta manera, la voz autoral del texto acaba influyendo en la decisión del TSJC, favoreciendo "el empleo de un determinado subconjunto de valores del sistema de valoración y desechando otros" (White, 2001: 9, trad. propia). En concreto, esta estrategia apunta a derrumbar el ethos de la querellada y el ideario soberanista $y$, por otro, otorgar carta de naturaleza a actitudes, creencias y supuestos que vertebran el discurso del constitucionalismo.

En cuanto acción social -a saber, proceder humano orientado por las acciones de otro (Weber, 1921) - la querella del fiscal se encuadra en un marco interactivo institucionalizado, definido por las convenciones del género discursivo. Según el acusador sea un procurador de los tribunales (Garofalo, 2009) o el ministerio fiscal, dicho marco interactivo admite variantes que tienen que ver con la organización de la escena de enunciación y con el reparto de los papeles discursivos entre los intervinientes. 
A partir del concepto de escena de enunciación, entendida como 'el interior del discurso', en el que la palabra es puesta en escena (Maingueneau, 1993), en la querella del fiscal cabe distinguir tres escenas distintas:

1. La escena englobante se refiere al tipo de escena general en el que hay que situarse para entender los propósitos retóricos y pragmáticos del emisor y de qué modo el destinatario es interpelado por el texto. En el caso que nos ocupa, la escena o evento englobante correspondería a presentar una queja ante un juzgado o tribunal.

2. La escena genérica es impuesta por el género discursivo específico: entre sus componentes, destacan los roles que desempeñan los participantes, y el propósito principal del emisor. Desde la perspectiva sociolingüística de Goffman (1981), el emisor actúa como autor de sus propios enunciados y, a la vez, como animador o reformulador de un punto de vista compartido por toda la Fiscalía Superior de Cataluña y por el Fiscal General del Estado. Este último órgano, valiéndose de su superioridad jerárquica requirió que se formulara querella contra Forcadell y, por tanto, desempeña en la interacción el papel de responsable. El destinatario, en cambio, se desglosa en destinatario directo (el TSJC, conocido, ratificado y apelado), para quien está específicamente construido el texto, y el destinatario indirecto, a saber, la presidenta del Parlament y los demás miembros independentistas del Govern. El principal objetivo del emisor consiste en constituirse en parte acusadora, solicitando al TSJC para que admita a trámite ambas querellas.

3. La escenografia, entendida como escena construida en el texto, legitima los enunciados seleccionados por el fiscal y permite la introducción de posturas evaluativas específicas para interpelar y persuadir al TSJC. Como tal, según Chareaudeau y Maingueneau (2002: 222), la escenografía no constituye el simple marco del texto, sino que remite a un esquema cognitivo concreto que anida y va consolidándose dentro de la escena genérica: "al emerger, la palabra implica una determinada escena de enunciación que, en realidad, se valida progresivamente a través de esa enunciación misma". A la luz de lo anterior, la escenografía activada por el fiscal al acusar a Forcadell y a los demás querellados es la de un 
panfleto ${ }^{1}$ de defensa de la Constitución de 1978 y del Estado de derecho, que legitima y fomenta las elecciones gramaticales y léxicas de carácter valorativo. En este sentido, recurriendo a las palabras de los precitados autores (2002: 222, cursiva añadida), la defensa apasionada del constitucionalismo, convertida en escenografía, es

aquello de lo que procede el discurso y lo que este discurso engendra: ella legitima un enunciado que a su vez debe legitimarla, debe dejar establecido que esa escenografía de la que procede la palabra es precisamente la escenografía requerida para contar una historia, denunciar una injusticia, etc.

Dentro de esta escenografía, las valoraciones específicas realizadas por el fiscal en las secuencias narrativas y argumentativas de ambos textos han de acomodarse a un enmarcado conceptual o guion -impuesto por los artículos 410.1 (delito de desobediencia) y 404 (delito de prevaricación continuada) del $\mathrm{CP}^{2}$ - definido por Taranilla (2012: 101) como "esquema temporalmente ordenado de acontecimientos y situaciones comunes". Basándose en Schank y Abelson (1987) y en la teoría del filósofo del derecho Nerhot (1990), la precitada autora destaca que la norma contiene un modelo que construye la realidad, asignando pertinencia a determinadas situaciones particulares y estandarizándolas, para que queden inmediatamente reconocibles por los interlocutores que poseen en su enciclopedia ese mismo esquema o enmarcado. El análisis cuantitativo y cualitativo que se ofrece a continuación demos-

\footnotetext{
${ }^{1}$ Se entiende aquí el término panfleto como "opúsculo de carácter agresivo" (DLE 2014, s.v.) o "folleto u hoja de propaganda política o de ideas de cualquier clase" (María Moliner 1992, s.v.), es decir, como discurso de apasionada defensa de los principios constitucionales.

${ }^{2}$ Los mencionados artículos tienen el siguiente tenor:

Art. 404.0: "A la autoridad o funcionario público que, a sabiendas de su injusticia, dictare una resolución arbitraria en un asunto administrativo se le castigará con la pena de inhabilitación especial para empleo o cargo público y para el ejercicio del derecho de sufragio pasivo por tiempo de nueve a quince años".

Artículo 410.1. "Las autoridades o funcionarios públicos que se negaren abiertamente a dar el debido cumplimiento a resoluciones judiciales, decisiones u órdenes de la autoridad superior, dictadas dentro del ámbito de su respectiva competencia y revestidas de las formalidades legales, incurrirán en la pena de multa de tres a doce meses e inhabilitación especial para empleo o cargo público por tiempo de seis meses a dos años".
} 
trará que los guiones específicos activados por los artículos del código penal anteriormente mencionados resultan determinantes para la selección de los elementos léxico-gramaticales de valor axiológico observables en ambos textos.

En efecto, la función acusadora del género querella hace que dichos elementos presenten una polaridad negativa y una carga afectiva tendente a la intensificación. Cuando quien formula la acusación es un procurador, según la gravedad del delito atribuido al querellado, se ha observado cierto grado de implicación de la subjetividad del emisor en las circunstancias personales de su cliente, unida a la manifestación de cierta empatía entre el procurador y su mandante (Garofalo, 2009: 175). Esta dinámica intersubjetiva no sorprende, ya que entre el abogado que redacta el texto y el querellante hay una relación profesional entre particulares, a raíz de la cual un profesional del derecho recibe un beneficio monetario para representar, con la debida contundencia, los intereses de su cliente. Un tanto distinto es el caso del subgénero "querella del Ministerio Fiscal", en el que quien se constituye en parte acusadora es una figura institucional llamada a actuar con mayor equilibrio e imparcialidad y a mantenerse equidistante de polémicas políticas, en cuanto defensora de la legalidad. Ahondando en esta línea, los epígrafes siguientes pondrán de manifiesto que la defensa del constitucionalismo desde los tribunales puede llegar a realizarse con una intensidad parecida al ímpetu de un ataque político.

\section{Metodología de análisis y objetivos de la investigación}

El presente estudio se inscribe en el marco de la investigación sobre la valoración y la emoción en los discursos especializados (López Ferrero, 2008; Diaz Rojo, 2010; Serpa, 2011, entre otros) y pretende integrar diferentes enfoques metodológicos, computacionales y semánticos, para lograr una comprensión más profunda de los recursos axiológicos movilizados por el ministerio fiscal. El recorrido analítico que se propone a tal efecto se articula en tres etapas interrelacionadas.

En primer lugar, se realiza una medición cuantitativa de las marcas de valoración presentes en ambas querellas, para que los elementos léxico-gramaticales que manifiestan la subjetividad del emisor "emerjan por sí solos" del corpus de estudio (Biber, 2009), proporcionando datos empíricos capaces de orientar el análisis. El primer estadio de 
esta investigación, por tanto, sigue un enfoque inductivo guiado por el corpus (corpus driven) y se basa en la medición de la polaridad y de la intensidad del sentimiento textual mediante la herramienta de análisis de sentimiento Lingmotif v.1.0 (Moreno-Ortiz, 2016). Entendido como procesamiento computarizado de la expresión de opiniones y juicios y emociones del emisor y, en general, de su subjetividad (Liu, 2010), el análisis de sentimiento (o minería de opinión) permite medir la carga afectiva de los textos y, pese a sus límites, ofrece la ventaja de cuantificar la fuerza de los ataques del fiscal con datos numéricos y de compararla con la intensidad del sentimiento en un corpus de referencia del género querella criminal.

Como se verá, los resultados arrojados por Lingmotif se basan en palabras valorativas aisladas, monolexémicas o polilexémicas, contenidas en el diccionario de la aplicación, configurado para la lengua estándar. Para paliar los inevitables errores de detección automática de la polaridad textual de elementos muy sensibles al contexto, se ha procedido a la constitución manual de un diccionario complementario específico (plugin), que recoge ítems léxicos axiológicos en el dominio de los delitos de desobediencia y de prevaricación.

La segunda etapa profundiza en el análisis de sentimiento e ilustra los criterios adoptados para seleccionar las marcas valorativas que se han incorporado al plugin, para que el vocabulario de complemento de Lingmotif no fuera un mero listado de palabras seleccionadas de manera impresionista. En concreto, se ha optado por un doble criterio semántico-estadístico, según el cual se han añadido al plugin solo palabras clave dotadas de frecuencia inusual (keywords), pertenecientes a los tres dominios de la Teoría de la Valoración (actitud, compromiso y gradación, véase Martin, 2000 y 2003; Martin y White, 2005; Martin y Rose, 2007). Para este fin, con la ayuda del programa AntConc (Anthony, 2014), se ha extraído la keyword list y se han observado las concordancias y los agrupamientos léxicos (clusters) de las palabras de dicha keyword list.

Por último, la tercera etapa de la investigación elabora los resultados de las dos primeras y propone un breve análisis cualitativo de cada uno de los tres dominios de la valoración en las dos querellas de referencia, combinando el enfoque basado en corpus y el guiado por el corpus (Tognini-Bonelli, 2001). El análisis cualitativo ofrece la ventaja de afinar el análisis de sentimiento y de profundizar en sus 
resultados, dado que las herramientas informáticas existentes no son capaces de interpretar adecuadamente las correlaciones semánticas de una información con otros conceptos aledaños y no siempre el analista dispone de modelos de representación del conocimiento especializado, p.ej., de ontologías o redes semánticas capilares del dominio penal, capaces de garantizar un análisis automatizado riguroso de los significados valorativos. Por tanto, se ha considerado necesario 'pulir' los resultados obtenidos con Lingmotif mediante una tasación cualitativa del sentimiento, considerando que la actitud del fiscal se manifiesta a menudo de forma implícita y no puede considerarse como una característica o propiedad de palabras individuales, sino de enunciados o textos enteros (White, 2001).

Cabe reconocer que este enfoque metodológico presenta tanto una limitación de fondo como una ventaja implícita. Por un lado, es inusual que una aplicación de Sentiment Analysis, concebida para el análisis automático de textos en lengua estándar, se aplique a un género judicial como la querella del Ministerio Fiscal y, en concreto, a un corpus de tamaño bastante reducido (solo 23.826 palabras). De hecho, la incorporación de un plugin lexicon tiende necesariamente a variar los valores de polaridad y, por esta razón, hubiera sido oportuno basar el análisis en un corpus suficientemente amplio, para determinar qué elementos lexicogramaticales se deben introducir en un plugin útil para el análisis automático de textos de este dominio penal.

Por otro lado, entendemos que la selección de los ítems axiológicos y la determinación de su polaridad son variables muy sensibles no solo a las dimensiones del corpus de estudio y al género textual, sino también al enmarcado cognitivo (escenografía y guiones delictivos) de los textos judiciales que se quieran analizar. El plugin constituido para esta investigación abarca solo palabras clave coherentes con el enmarcado concreto impuesto por el fiscal y, por esta razón, creemos que los resultados obtenidos - pese a sus límites- pueden resultar significativos para el tratamiento automático de otros textos dotados del mismo frame, orientado hacia la defensa apasionada del constitucionalismo y hacia la reprobación de conductas desobedientes y prevaricadoras, actualmente imputadas a más de una figura del independentismo por los jueces de Cataluña.

En resumen, el estudio propuesto constituye un caso de triangulación (McNeill, 1990: 22), ya que intenta conjugar múltiples enfoques 
metodológicos, discursivos y computacionales, para ofrecer una descripción empíricamente cimentada de las marcas de subjetividad del fiscal, en cuya voz el discurso judicial se hibrida con el político.

\section{Medición de la polaridad de las querellas con Lingmotif $v$. 1.0}

El grado de implicación subjetiva del fiscal en ambas querellas se ha cuantificado, de entrada, mediante el software Lingmotif (MorenoOrtiz, 2016), una aplicación de análisis de sentimiento capaz de identificar en los textos palabras y frases con carga afectiva, contenidas en los diccionarios del programa, y de aplicar reglas de contexto (de inversión, intensificación y atenuación), para dar cabida a posibles modificadores del sentimiento (Moreno-Ortiz, 2017: 133).

Los valores arrojados por Lingmotif se diferencian en dos magnitudes, a saber, el TSI (Text Sentiment Intensity) o índice de intensidad del sentimiento textual -es decir, la relación entre ítems que expresan sentimiento e ítems de valor no emocional-y el TSS (Text Sentiment Score), o valor global del sentimiento textual, expresado como promedio de elementos positivos, negativos y neutros contenidos en cada texto. Ambas magnitudes se miden en una escala graduada, concebida como un continuum de valores de 0 a 100 , que van, para el TSS, de lo extremadamente negativo $(<20)$ a lo extremadamente positivo $(>80)$ y, para el TSI, de lo extremadamente factual $(<55)$ a lo extremadamente intenso $(>85)$. El programa asigna una valencia positiva (entre 5 y 2 ), negativa (entre -5 y -2) o neutra a cada ítem léxico (excepto a las palabras gramaticales) y los valores del TSI reflejan el porcentaje de las valencias asignadas, teniendo presente la longitud de cada texto.

Se ha realizado un análisis conjunto de las dos querellas (23.826 palabras en total), ya que ambas apuntan a enjuiciar delitos idénticos cometidos por las mismas personas, con una cronología y una dinámica ligeramente diferente. La decisión de reunir ambos textos en un único corpus de análisis se debe también a razones funcionales y estructurales. En primer lugar, la propia Fiscalía solicita la acumulación de la segunda querella a las diligencias previas activadas por la primera y seguidas ante el TSJC. En segundo lugar, se han comparado los dos textos con la herramienta de traducción asistida por ordenador SDL Trados Studio y este cotejo ha evidenciado que la primera querella presenta un 
total de 9.587 palabras y la segunda un total de 14.239 palabras, de las cuales 7.649 están tomadas y repetidas del primer texto.

Cabe destacar que el diccionario de Lingmotif (que incluye, para el español, 207.000 palabras y 300 reglas contextuales, Moreno-Ortiz, 2017: 137) está concebido para analizar el sentimiento de textos de registro estándar, aunque permite utilizar léxicos específicos, elaborados por el propio usuario a modo de léxico complementario (plugin), lo cual posibilita el análisis de la carga afectiva de géneros especializados. Dado que la orientación semántica depende del ámbito de especialidad (Moreno-Ortiz \& Fernández Cruz, 2015: 332), a falta de un extractor estadístico capaz de identificar con cierta fiabilidad candidatos a términos de un corpus de querellas, el análisis ha requerido la elaboración manual de un plugin específico, capaz de detectar la polaridad del léxico de la Fiscalía en ambos documentos. Los criterios para la constitución del plugin se detallan en el epígrafe siguiente; lo que interesa destacar aquí es que, de entrada, se ha efectuado un análisis del sentimiento de ambos textos, con y sin diccionario de complemento, lo que ha producido los siguientes resultados:
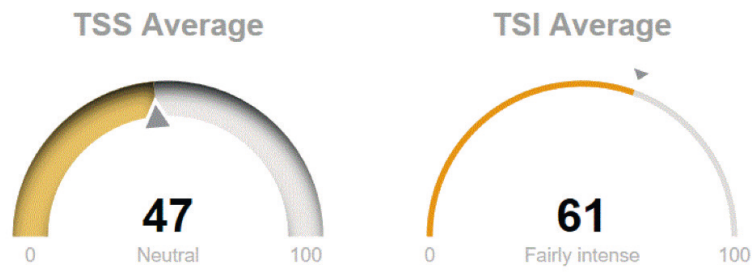

Fig. 1. Análisis de sentimiento de ambas querellas sin plugin
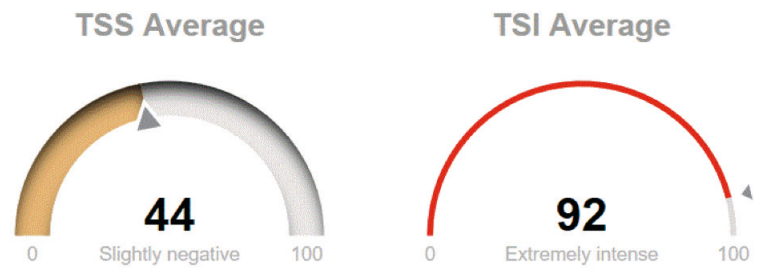

Fig. 2. Análisis de sentimiento de ambas querellas con un plugin específico 
Lo que se observa a simple vista es que, tras incorporar el léxico específico, la aplicación arroja valores bastante parecidos de TSS (con un ligero viraje de lo neutro a lo ligeramente negativo) e índices de TSI muy distintos, hasta alcanzar un índice extremadamente intenso (92) del sentimiento global en el segundo análisis (fig. 2).

Para interpretar correctamente estos datos, relacionándolos con el género de estudio, se ha procedido al cálculo de la línea de base, es decir, de los valores que expresan la normalidad estadística del TSS y del TSI en un corpus de referencia de 63 querellas formuladas por un amplio abanico de delitos (629.893 palabras en total):
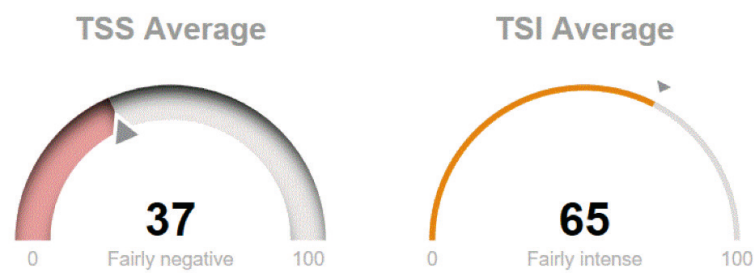

Fig. 3. Línea de base del TSS y del TSI en el corpus de referencia

Los indicadores de la figura 3, recabados a partir del diccionario de Lingmotif, demuestran que el género querella, cuya función primaria consiste en mover una acusación contra alguien, suele caracterizarse por un léxico de polaridad bastante negativa y por una carga afectiva tendiente a la intensificación. Según la gravedad del delito atribuido al querellado, la implicación de la subjetividad del procurador en las circunstancias personales de su cliente, unida a la manifestación de cierta empatía entre animador y autor del texto, son estrategias discursivas ya observadas en la querella española y, en general, ausentes en los textos paralelos italianos (Garofalo, 2009: 175). No es baladí advertir que, en las 63 querellas del corpus de referencia, entre el procurador que redacta el texto y el querellante hay una relación profesional entre particulares, en la que el abogado recibe un beneficio monetario para representar, con la debida contundencia, los intereses de su cliente. Un tanto distinto es el caso que nos ocupa, en el que quien se constituye en parte acusadora es el Ministerio Fiscal, figura institucional que actúa como defensor de la legalidad y que suele intervenir con mayor equilibrio y 
con la imparcialidad que corresponde (o debería corresponder) a sus funciones. De ahí que el promedio de elementos léxicos positivos, negativos y neutros (TSS), en ambos textos de la Fiscalía General de Cataluña (fig. 1), tienda a una mayor neutralidad, resultando cuatro puntos superior a la línea de base (fig. 3). Ello no significa, no obstante, que el Fiscal defienda su tesis con menor dureza, como parece demostrar el valor bastante intenso del TSI (fig. 1), apenas cuatro puntos inferior a la línea de base.

Es oportuno destacar que los resultados comentados hasta aquí no varían de forma significativa si se repite el análisis de sentimiento del corpus de referencia incorporando el plugin específico elaborado para las dos querellas contra Forcadell: se obtiene un mero incremento de un punto del valor del TSS (38), mientras que el índice de TSI baja una unidad (64). Este resultado se interpreta fácilmente, ya que el plugin funciona para detectar la carga afectiva del léxico relacionado con los delitos de desobediencia y prevaricación, y el corpus de referencia no contempla casos subsumibles en la misma tipificación jurídica.

Por último, es interesante señalar que, tras analizar el corpus de cotejo con Lingmotif, dos textos presentan un índice de TSI igual a 100: se trata, respectivamente, de una querella formulada por un delito que vio al Partido Popular como parte ofendida y de otra presentada por delitos que se produjeron a raíz de un proyecto de ley impulsado por el mismo partido $^{3}$. Ligeramente por debajo del valor máximo de TSI, en el corpus de referencia, destacan una querella por injurias presentada por el lehendakari Ibarretxe contra un periodista de El País $($ TSI $=97)$ y otra formulada por algunos ciudadanos españoles contra el expresidente del Gobierno del Partido Popular José María Aznar, por delitos contra personas y bienes protegidos en caso de conflicto armado $(\mathrm{TSI}=94)$. Los datos cuantitativos parecen indicar, por tanto, que el sentimiento textual global se hace acusadamente intenso cuando el discurso judicial y el político se hibridan, en textos ideológicamente polarizados.

\footnotetext{
${ }^{3}$ En el primer caso, se trata de una querella presentada (por delitos de injurias) por la asociación Tertulia Feminista 'Les Comadres' contra el obispo de Alcalá de Henares, a raíz de una protesta contra la reforma de la Ley de Aborto, impulsada por el Ministro de Justicia Alberto Ruiz Gallardón. En el segundo caso, el querellante es el Partido Popular contra el secretario general de la Federación Socialista Madrileña, por los delitos de injurias, calumnias, coacciones y amenazas.
} 


\section{Constitución del plugin y análisis de los términos seleccionados}

La selección de los 328 términos de polaridad positiva o negativa relacionados con los delitos de desobediencia y prevaricación e incluidos en el plugin se ha realizado manualmente, tras una atenta lectura de ambos textos. Pese al margen de error que todo análisis manual entraña, la constitución del plugin se ha ajustado al siguiente enfoque híbrido, a la vez estadístico y semántico:

1. Creación de una lista de palabras clave ordenadas por valor de keyness ("calidad de palabra clave");

2. Identificación de términos monolexémicos y polilexémicos de polaridad positiva y negativa, según un criterio onomasiológico. Observación de las concordancias de los términos clave y de sus respectivos colocados y clusters;

3. Comprobación de que los términos identificados figuren en la lista de palabras clave y que no estén ya incluidos en el diccionario de Lingmotif. Determinación de la polaridad que dichos ítems asumen en la lengua común y en el ámbito penal;

4. Clasificación del léxico evaluativo obtenido en cuatro subgrupos: a) términos que manifiestan carga afectiva hacia las conductas supuestamente ilícitas de los querellados; b) términos que evalúan productos normativos (p. ej., resoluciones aprobadas por la Cámara catalana, derivadas de las conductas criminógenas); c) recursos léxicos y gramaticales por medio de los cuales la voz del Fiscal se posiciona intersubjetivamente (p. ej., estructuras polifónicas, verbos modales, negaciones y elementos evidenciales); c) valoraciones escalares. Todos los elementos axiológicos identificados de esta manera han resultado compatibles con la escenografía del discurso y con los guiones de los delitos de desobediencia y de prevaricación continuada $(\S 1)$.

El primer estadio de la metodología antedicha consiste, por tanto, en la extracción de las 1965 palabras relevantes de ambas querellas, obtenidas mediante la función Keyword list del programa AntConc (Anthony, 2014), que compara los dos textos de la Fiscalía Superior de Cataluña con el corpus de referencia. De dichas palabras, se han eliminado los ítems léxicos semánticamente vacíos (palabras gramaticales) 
y los acrónimos característicos del ámbito judicial de referencia (p. ej., LOTC, Ley Orgánica del Tribunal Constitucional, CE, Constitución española, etc.). Tras esta operación de limpieza, las primeras 50 palabras clave de ambas querellas, ordenadas por índice de keyness, resultan ser las siguientes:

\begin{abstract}
Cataluña, parlamento, constituyente, resolución, constitucional, proceso, Constitución, presidenta, desobediencia, votación, estudio, parlamentaria, mandatos, referéndum, incidente, paralizar, resoluciones, tribunal, mandato, poderes, catalán, desconexión, pleno, parlamentarios, parlamentarias, cumplimiento, propuestas, boletín, parlamentario, julio, eludir, Carme, Forcadell, suponga, ordenamiento, ignorar, voluntad, parlament, providencia, creación, inviolabilidad, democrático, iniciativa, conclusiones, inconstitucional, impugnación, negativa, decisiones, suspensión, soberanía
\end{abstract}

Según lo previsto, la lista así obtenida contiene dos clases de palabras (Baker 2006: 127): nombres propios que identifican el marco espacio-temporal, el dominio del discurso (en concreto, la escenografía y los guiones delictivos descritos en $\S 1$ ) y la protagonista principal de los hechos encausados (Cataluña, junio, Constitución, Carme, Forcadell), más una serie de palabras clave relacionadas con la temática central (aboutness keywords). A partir de estas últimas, analizando las concordancias de cada una de ellas, sus colocados y los clusters a la derecha, se evalúa la polaridad efectiva de los candidatos a términos que se incluirán en el plugin.

La clasificación semántica de estos términos clave se ha realizado acudiendo a los tres dominios de la teoría de la valoración (Martin, 2000, 2003; Martin \& Rose, 2007; White, 2001, 2003; Martin \& White, 2005), es decir, la actitud, el compromiso y la gradación. Como es sabido, en la actitud se incluyen los significados mediante los cuales el emisor atribuye un valor o una evaluación intersubjetiva al comportamiento de los querellados en relación con las normas penales y a los productos de sus respectivas actuaciones. En el dominio del compromiso se incluyen los recursos lingüísticos utilizables para posicionar la voz del fiscal en relación con las diversas proposiciones o iniciativas de los partidos independentistas mencionadas en el texto. Por último, por medio de la gradación se representa un espacio semántico de escala relacionado con 
la manera en el que el fiscal intensifica o atenúa la fuerza de sus enunciados o gradúa el foco de sus categorizaciones semánticas.

Un análisis exhaustivo de todos y cada uno de los términos seleccionados para el plugin excedería con creces los límites de espacio de este estudio; los epígrafes siguientes se limitarán, por tanto, a ilustrar algunos casos representativos para cada dominio semántico.

\section{1. Ítems léxicos que manifiestan actitud}

En el marco del dominio de la actitud, la Fiscalía se limita a evaluar el comportamiento de los querellados (subdominio del juicio) y los productos de su actuación, a saber, el proceso de desconexión y la producción normativa del Parlament, encaminada a llevar a cabo el referéndum vinculante en Cataluña (subdominio de la apreciación).

Por lo que se refiere al ámbito del juicio, desde la perspectiva del fiscal constituyen delito y se cargan de valoración negativa una serie de comportamientos que, ignorando las repetidas advertencias del TC, infringen el art. 410.1 (desobediencia) y el art. 404 (prevaricación continuada) del CP. No sorprende, pues, que en la lista de palabras clave desobediencia (índice de keyness 186.754) figure inmediatamente después de presidenta (keyness 200.417). En efecto, es suficiente observar los clusters en un intervalo de 15 palabras a la derecha del nombre y apellido de la presidenta para encontrar valoraciones contundentes (señaladas en cursiva a continuación) como:

La Sra. Presidenta del Parlamento de Cataluña, Carme Forcadell i Lluís, manifestando una voluntad inequivoca e irreversible de llevar adelante su proyecto político por la fuerza de los hechos consumados, con total desprecio de la Constitución de 1978, del ordenamiento emanado de la misma, y de los pronunciamientos de la STC de 2 de diciembre de 2015 y del ATC de 19 de Julio de 2016, procedió a dar impulso al proceso constituyente preordinado en la Resolución 1/XI.

La conducta de Doña Carme Forcadell que con su voto permitió el debate y votación de las propuestas registradas con los números [...] evidencia aún más su contumaz y obstinada voluntad de incumplir los mandatos constitucionales [...].

Repárese en que los comportamientos delictivos de la querellada se expresan mediante elementos léxicos que, en la lengua estándar, no 
tienen carga afectiva alguna (p. ej., permitir el debate y la votación, llevar adelante su proyecto político) y que, desde la perspectiva soberanista, constituyen la quintaesencia del derecho a decidir de la Cámara catalana. Dichas expresiones, sin embargo, se han incluido en el plugin no solo porque constituyen las acciones criminógenas esenciales que motivan las querellas, sino también porque coaparecen junto a modalizadores adjetivos y adverbiales de inequívoca polaridad negativa, que vehiculan la reprobación más severa del fiscal (voluntad inequívoca e irreversible, aún más, con total desprecio, por la fuerza de los hechos consumados, su contumaz y obstinada voluntad).

Se ha observado, además, que en la mayoría de las concordancias la palabra clave voluntad se refiere a los propósitos de los querellados y, por tanto, presenta una prosodia semántica acusadamente negativa (p. ej., voluntad obstativa, rebelde, de incumplir los mandatos, de desobedecer, de no dar cumplimiento a las decisiones, etc.). Del mismo modo, el adverbio constitucionalmente se emplea casi siempre para evaluar críticamente la conducta de Forcadell (ser constitucionalmente ilegítimo, ilícito; no resultar constitucionalmente admisible) y contribuye a la creación de la metáfora conceptual de fondo (Lakoff \& Johnson, 2003) "el soberanismo rompe la legalidad".

Si las palabras con el índice más elevado de keyness representan los nudos temáticos de ambos textos y expresan significados ideacionales, las menos frecuentes pueden encapsular significados connotativos o metafóricos interpersonales. Por ej., el sustantivo ardid, los adjetivos camuflada [retórica] y voluntarioso, el verbo enmascarar o el adverbio torticeramente, que ocupan respectivamente el lugar 1.136, 684, 428, 599 y 1041 de la keyword list, contienen metáforas lexicalizadas que descalifican el ethos de la querellada y ponen en entredicho su honradez institucional:

Son estos actos de la Presidencia, utilizando torticeramente el Reglamento de la Cámara, los que lesionan el bien jurídico.

[Forcadell sustituye la ejecución de la sentencia del TC] por un voluntarioso intercambio de argumentos con los que enmascarar la conducta desobediente [...].

El pretendido ardid elucubrado para evitar la intervención de la Mesa y trasladar la eventual responsabilidad a un Pleno irresponsable no es sino una camuflada retórica al servicio del incumplimiento. 
En varias ocasiones ha sido necesario invertir la polaridad asignada por defecto por Lingmotif a algunos elementos léxicos que expresan sentimiento, p. ej., a la expresión ardid elucubrado. De hecho, el programa atribuye una polaridad positiva a cualquier sujeto lógico de un verbo implicativo como evitar (Sbisà, 2007: 59-62), que suele activar la presuposición de que la consecuencia evitada es mala y la causa que la evita es buena.

El subdominio de la apreciación, en cambio, abarca el conjunto de evaluaciones sobre los 'productos' del Parlament (p. ej., la Resolución 1/XI del Parlamento de Cataluña, sobre el inicio del proceso político en Cataluña) o sobre el proceso de desconexión. Nótese que tanto resolución como proceso son palabras con un valor de keyness muy elevado (526.149 y 421.294), en cuyos clusters (a la derecha) figuran elementos léxicos que manifiestan una actitud censoria palmaria:

La resolución [...] no es efecto de una aplicación de la Constitución, sino pura y simplemente, producto de [la] libertad [del Parlament], convertida irrazonablemente en fuente de norma particular.

$\mathrm{Al}$ ratificar y asumir como propias las conclusiones aprobadas por la referida comisión parlamentaria, el Parlamento de Cataluña elude los pronunciamientos de la STC 259/2015 e ignora las advertencias del ATC 141/2016, pues pretende dar continuidad y soporte al denominado "proceso constituyente en Cataluña" dirigido a su desconexión del Estado español.

El análisis de las concordancias revela que resolución y proceso, palabras neutras en español estándar, se cargan de valor negativo, evidenciando una marcada preferencia semántica por relacionarse con elementos léxicos que remiten a conductas improcedentes o ilegales (irrazonablemente, eludir, ignorar, pretender, desconexión).

\section{2. Ítems léxicos que expresan compromiso}

La semántica del compromiso presupone una interpretación heteroglósica de ambos textos de la Fiscalía, cuyo andamiaje argumentativo se construye a partir de la voz del oponente, con la que el emisor polemiza, en una continua tensión dialéctica. Desde una perspectiva polifónica e interaccionista, al recurrir a un verbo modal como deber, el fiscal no pretende solo expresar un significado lógico-deóntico, sino que mani- 
fiesta también rechazo y hostilidad hacia la postura de los querellados. Nótese, p. ej., cómo el modal debe se revela útil para acometer contra la postura de Forcadell, quien apela a su inviolabilidad e invoca una interpretación elástica de la Ley:

La STC n ${ }^{\circ} 51 / 1985$, de 10 de abril, estableció que todo lo que afecta a las prerrogativas parlamentarias debe ser interpretado de forma estric$t a$, no cubriendo la inviolabilidad cualquier actuación, aún con relevancia política, del parlamentario.

Así pues, si las frecuentes citas directas de la jurisprudencia del Tribunal Constitucional o del Tribunal Supremo funcionan como mecanismos de respaldo de la tesis defendida, el punto de vista de los querellados puede evaluarse y neutralizarse de forma más indirecta.

Siguiendo con el análisis del significado interpersonal de los verbos modales, se observa que poder aparece con altísima frecuencia (en 19 de las 26 ocurrencias totales) en contrargumentaciones que apuntan a la total indisponibilidad del fiscal para negociar con la opinión del contrincante. Por esta razón, suele coligarse con el adverbio de negación no o con palabras de polaridad negativa (en ningún caso):

No puede alegarse para negar la desobediencia que la querellada o sus asesores llegaran a la conclusión de que lo realizado no incumplía las providencias del Tribunal Constitucional [...].

La inviolabilidad no puede concebirse como cobijo de la arbitrariedad, sino que los actos parlamentarios quedan sometidos a la Constitución española.

El ordenamiento jurídico, con la Constitución en su cúspide, en ningún caso puede ser considerado como límite de la democracia, sino como su garantía misma.

La relevancia semántica de estas negaciones queda comprobada no solo por la presencia entre sus constituyentes de keywords como alegarse, concebirse o considerado (que ocupan respectivamente el lugar 1328, 218 y 1299 de la lista de palaras clave) sino también por su proximidad con conceptos nucleares expresados por palabras con un valor de keyness más elevado, p. ej., desobediencia, constitución, inviolabilidad, ordenamiento, que resultan totalmente coherentes con los guiones delictivos activados por el fiscal y figuran entre las 50 primeras palabras clave. 
Asimismo, evocan y evalúan negativamente la voz del oponente algunos elementos lexicogramaticales de valor evidencial y ciertos recursos ortográficos como el entrecomillado (p. ej., "el denominado "proceso constituyente" en Cataluña", "una supuesta legitimidad democrática", "el pretendido ardid elucubrado para evitar la intervención de la Mesa").

Los elementos valorativos mediante los cuales el fiscal alude a la postura de los querellados son, en su aplastante mayoría, "proclamaciones", es decir, enunciados implícitamente polifónicos mediante los cuales el emisor aumenta la fuerza de su compromiso con el contenido proposicional de sus aseveraciones. Se trata de una opción de "intravocalización cerrada" (White, 2001: 25), que evoca la voz del oponente para desacreditarla y suprimirla, limitando las posibilidades de interacción con la diversidad ideológica:

El texto constitucional refleja las manifestaciones del principio democrático, cuyo ejercicio no cabe fuera del mismo [STC 42/2014]. Por ello, el ordenamiento jurídico, con la Constitución en su cúspide, en ningún caso puede ser considerado come límite de la democracia, sino como su garantía misma (FJ 50). [...].

\subsection{Valores que indican gradación}

Las valoraciones expresadas mediante una escala de grado apuntan a enfatizar la fuerza interpersonal que el fiscal atribuye a sus enunciados o bien agudizan el foco de sus valoraciones. La amplificación de la carga afectiva se logra, p. ej., mediante los adverbios focales o mensurativos en -mente (Pinuer Rodríguez y Oteíza Silva, 2015: 112-116). Los focales (p. ej., estrictamente, precisamente, meramente, etc.) explicitan que la entidad individuada está jerarquizada entre varias posibles y establecen una relación "entre su foco y el conjunto de alternativas posibles con las que se contraponen expresa o tácitamente" (NGLE, 2009: 2992). El adverbio que agudiza el foco de la valoración con el mayor índice de keyness (8.464) es claramente: aparece 10 veces y presenta una prosodia negativa en 9 casos (contravenir los mandatos, adoptar acuerdos contrarios, lesionar el bien jurídico, desbordar los estrechos márgenes de la excusa absolutoria). El significado interpersonal de este elemento es el de 'estrechar el foco de la evaluación', adscri- 
biendo los hechos narrados a conductas típicas previstas y sancionadas en el código penal.

Los adverbios mensurativos, en cambio, son cuantificadores escalares o presuposicionales que acrecen la fuerza del posicionamiento intersubjetivo, ya que se forman a partir de adjetivos axiológicos (absoluto, sobrado). La cuantificación que expresan sitúa un elemento dentro de un conjunto, donde se diferenciará por su posición escalar, que suele establecerse a partir de factores pragmáticos, dependientes de la subjetividad del emisor. El fiscal opta por mensurativos que señalan el máximo grado de la escala negativa, entre los cuales, en la keyword list, aparecen, p. ej., absolutamente (0.742) y sobradamente (0.953):

La actividad de la comisión creada resulta absolutamente inviable si no se entiende condicionada al cumplimiento de las exigencias de la Constitución.

La resolución 1/XI [...] excede sobradamente de los límites que [el TC] imponía a la Comisión de Estudio.

Como puede observarse, el principio de gradación de fuerza opera intrínsecamente en los valores de actitud, en el sentido de que cada significado actitudinal representa un punto específico en una escala de intensidad de menor a mayor. Para constituir el plugin, a los términos que identifican escuetamente una conducta delictiva (p. ej., celebrar el referéndum, ejecutar la acción típica, permitir la alteración del orden del día) se les ha asignado una valencia -2, mientras que a los ítems que manifiestan el máximo grado de reprobación o el máximo riesgo para el ordenamiento constitucional (con total desprecio de la Constitución, creación de un Estado catalán) se les ha atribuido una valencia -5.

\section{Conclusiones}

Se ha ofrecido un análisis cuantitativo y cualitativo del léxico del sentimiento manifestado por la Fiscalía General de Cataluña en las dos querellas contra Carme Focadell i Lluís, presidenta del Parlamento catalán. A simple vista, los datos cuantitativos recabados con la aplicación Lingmotif indican que en ambos textos el fiscal manifiesta una carga afectiva de cierta intensidad, con un valor de TSI (61) parecido a la línea de base (65) calculada en un corpus de referencia de 63 querellas (629.893 palabras en total). Profundizando más en el análisis semántico de los ele- 
mentos lexicogramaticales, descubrimos que el vocabulario de Lingmotif, programado para el análisis de la lengua estándar, no detecta varios ítems léxicos de polaridad negativa que jalonan la argumentación del fiscal. Por consiguiente, ha sido necesario constituir un vocabulario de complemento (plugin) específico, que la aplicación permite incorporar. Los términos monolexémicos y polilexémicos incluidos en el plugin se han seleccionado manualmente, teniendo en cuenta su coherencia con el enmarcado cognitivo del texto (escenografía y guiones delictivos) y su calidad de palabra clave (keyness), sistematizando su valor axiológico según los tres dominios semánticos previstos por la teoría de la valoración (actitud, compromiso y gradación). Tras la incorporación del plugin específico, se ha obtenido un valor de intensidad del sentimiento (92) sorprendentemente elevado, afín al índice de TSI que presentan algunas querellas del corpus de referencia, en las que el discurso judicial se hibrida con el político, lo cual parece indicar que ambas querellas contra Forcadell son ejemplos de politización de la justicia.

Pese a la brevedad del corpus de estudio, la constitución del plugin ha permitido cuantificar la carga valorativa de ítems léxicos que presentan una polaridad neutra en la lengua común, pero que cobran un significado negativo evidente en la escenografía y en el guion activados por el fiscal (p. ej., permitir el debate y la votación; llevar adelante un proyecto político, proceso constituyente en Cataluña, etc.). De este modo, ha sido posible integrar la dimensión cognitiva específica de este dominio penal al análisis de sentimiento realizado con Lingmotif. Por otra parte, el análisis cualitativo ha resultado esencial para seleccionar los ítems incluidos en el plugin según un criterio semántico-funcional. En concreto, las tres categorías que vertebran la Teoría de la Valoración han ofrecido la pauta de clasificación de los elementos del léxico complementario, posibilitando, p. ej., la inclusión de elementos que definen no solo la conducta ilícita de los querellados, sino también el posicionamiento intersubjetivo del fiscal (valores de negación y de contraargumentación, entre otros), los valores de gradación y los enunciados implícitamente polifónicos.

El trabajo de extracción de los candidatos a términos se hubiera podido intentar con la ayuda de un extractor estadístico, basado en algoritmos de aprendizaje automático que comparan las frecuencias de las palabras de un dominio específico y de un corpus general, compaginando mediciones estadísticas con varias técnicas heurísticas (Moreno- 
Ortiz \& Fernández-Cruz, 2015: 333). Es bastante probable, sin embargo, que este enfoque estadístico no hubiera logrado resultados del todo satisfactorios, ya que, como se ha observado, la mayoría de los ítems incorporados al plugin pertenecen al vocabulario semitécnico, formado "por unidades léxicas del lenguaje común que han adquirido uno o varios nuevos significados dentro del español jurídico" (Alcaráz Varó \& Hughes, 2002: 59) mediante un proceso de resemantización. Asimismo, la carga afectiva asociada a los recursos lexicogramaticales analizados parece depender en gran medida del contexto argumentativo de uso. De ahí la necesidad de elaborar ontologías jurídicas cada vez más afinadas que permitan la gestión automática y el análisis de sentimiento de documentos procesales.

\section{Bibliografía}

Alcaraz Varó, Enrique \& Hughes, Brian. 2002. El español jurídico. Barcelona: Ariel Derecho.

Baker, Paul. 2006. Using Corpora in Discourse Analysis. London/New York: Continuum.

Biber, Douglas. 2009. A corpus-driven approach to formulaic language: Multi-word patterns in speech and writing. International Journal of Corpus Linguistics 14: 275-311.

Charaudeau, Patrick \& Maingueneau, Dominique. (2002) 2005. Diccionario de análisis del discurso. Buenos Aires: Amorrortu.

Díaz Rojo, José Antonio. 2010. El lenguaje valorativo en noticias periodísticas españolas sobre avances médicos. Tonos 20. https://www.um.es/tonosdigital/znum20/secciones/estudios-5-el_lenguaje_valorativo_en_noticias.htm [Acceso 10/08/2017].

Garofalo, Giovanni. 2009. Géneros discursivos de la justicia penal. Milano: FrancoAngeli.

Goffman, Erving. 1981. Forms of Talk. Philadelphia: University of Pennsylvania Press.

Halliday, M.A.K. \& Hasan, Ruqaiya. 1985. Language, Context and Text: Aspects of Language in a Social-Semiotic Perspective. Oxford: Oxford University Press.

Lakoff, George \& Johnson, Mark. 2003. Metaphors we live by. Chicago/London: The University of Chicago Press.

Liu, Bing. 2012. Sentiment Analysis and Opinion Mining. San Rafao, CA: Morgan \& Claypool Publishers. 
López Ferrero, Carmen. 2008. La valoración y la emoción en español en discursos especializados. En Moreno Sandoval, Antonio (ed.) El valor de la diversidad (meta)lingüistica: Actas del VIII congreso de Lingüistica General. http://www.lllf.uam.es/clg8/actas/index.html [Acceso 10/08/2017].

Maingueneau, Dominique. 1993. Le contexte de l'oeuvre littéraire. Énonciation, écrivain, société. Paris: Duunod

Martin, James R. \& Rose, David. 2007. Working with Discourse. London/New York: Continuum.

Martin, James R. \& White, Peter R.R. 2005. The Language of Evaluation, Appraisal in English. London/New York: Palgrave Macmillan.

Martin, James R. 2000. Beyond Exchange: APPRAISAL Systems in English. En Hunston, S. \& Thompson, G. (eds), Evaluation in Text. Oxford: Oxford University Press.

Martin, James R. 2003. Introduction. Text 23(2): 171-181.

McNeill, Patrick. 1990. Research Methods. London: Routledge.

Moreno-Ortiz, Antonio \& Fernández-Cruz, Javier. 2015. Identifying polarity in financial texts for sentiment analysis: a corpus-based approach. Procedia. Social and Behavioral Sciences 198: 330-338.

Moreno-Ortiz, Antonio. 2017. Lingmotif: A User-focused Sentiment Analysis Tool. Procesamiento del Lenguaje Natural, Revista 58: 133-140.

Nerhot, Patrick. 1990. The law and its reality. En Nerhot, P. (ed.) Law, interpretation and reality. Dordrecht/Boston/London: Kluwer, 50-69.

Pinuer Rodríguez, Claudio \& Oteíza Silva, Teresa. 2015. Los adverbios en -mente como factor de valoración en el discurso de la historia. Verba 42: 99-134.

Real Academia Española \& Asociación de Academias de la Lengua Española. 2009. Nueva Gramática de la lengua española. Madrid: Espasa. (NGLE).

Sbisà, Marina. 2007. Detto e non detto. Roma/Bari: Laterza.

Schank, Roger \& Abelson, Robert. 1987. Guiones, planes, metas y entendimiento: un estudio de las estructuras del conocimiento humano. Barcelona: Paidós [1977].

Serpa, Cecilia. 2011. Significados interpersonales en los géneros legislativos: el texto como macropropuesta. Pragmalingüística 19: 96-114.

Taranilla, Raquel. 2012. La Justicia Narrante. Cizur Menor: Aranzadi.

Tognini-Bonelli, Elena. 2001. Corpus Linguistics at Work. Amsterdam/Philadelphia: John Benjamins.

Weber, Max. (1921) 1977. Economía y sociedad. México: Fondo de Cultura Económica. 
Persiguiendo con imparcialidad "el total desprecio a la Constitución”... 103

White, Peter R.R. 2001. An introductory tour through appraisal theory. The Appraisal Website. http://www.grammatics.com/appraisal/ [Acceso 29/03/2017].

White, Peter R.R. 2003. Beyond modality and hedging: A dialogic view of the language intersubjective stance. Text 23(2): 259-284.

Anthony, Laurence. 2014. AntConc (Version 3.4.3) [Computer Software]. Tokyo: Waseda University. http://www.laurenceanthony.net/

Moreno-Ortiz, Antonio. 2016. Lingmotif 1.0 [Computer Software]. Málaga: Universidad de Málaga. http://tecnolengua.uma.es/lingmotif 
